\title{
ON THE STABILITY OF SOLUTIONS TO CONFORMABLE STOCHASTIC DIFFERENTIAL EQUATIONS
}

\author{
GUANLI XIAO AND JINRONG WANG
}

Received 29 February, 2020

\begin{abstract}
In this paper, we study the stability of solutions to conformable stochastic differential equations. Firstly, we show the trivial solution are stochastially stable, stochastically asymptotically stable and almost surely exponentially stable, respectively. Secondly, we show the nontrivial solution are Ulam's type stable in the sense of probabilities. Finally, two examples are given to present the theoretically results.
\end{abstract}

2010 Mathematics Subject Classification: $60 \mathrm{H} 10$

Keywords: stochastic differential equations, conformable derivative, stability

\section{INTRODUCTION}

Conformable derivative, an extension of the classical limit definitions of the derivatives of a function, has been proposed in Khalil et al. [1]. The details of the basic theory are reported in [2,4] and the application are reported in [3,9]. The theory of stochastic differential equations (SDE) and Itô integrals have been studied extensively, one can refer to the classical results in $[5,6]$.

Consider the stability of solutions to the following conformable stochastic differential equations

$$
\left\{\begin{aligned}
D_{\rho}^{\alpha} X(t) & =b(X(t), t)+\sigma(X(t), t) \frac{d W(t)}{d t}, \quad \rho \in(0,1], t \in[\alpha, \infty), \\
X(\alpha) & =X_{\alpha}
\end{aligned}\right.
$$

where $D_{\rho}^{\alpha}$ is conformable derivative, $b: \mathbb{R}^{n} \times[\alpha, \alpha+h] \rightarrow \mathbb{R}^{n}, h>0$, and $\sigma: \mathbb{R}^{n} \times$ $[\alpha, \alpha+h] \rightarrow \mathbb{R}^{n \times m}$ are continuous and $W(\cdot)$ is a standard scalar Brownian motion on an underlying complete filtered probability space $\left(\Omega, \mathcal{F}, \mathbb{F}:=\left\{\mathcal{F}_{t}\right\}_{t \in[\alpha, \infty)}, P\right) . \mathcal{F}$ is a $\sigma$-algebra and $P$ is a probability measure. For each $t \in[\alpha, \infty), \mathbb{L}^{2}\left(\Omega, \mathcal{F}_{t}, P\right)$ denotes the space of all $\mathcal{F}_{t}$-measurable, mean square integrable functions endowed with the standard norm.

This work was supported in part by the Major Research Project of Innovative Group in Guizhou Education Department ([2018]012). 
Existence and uniqueness of solution to (1.1) is given in [7] by using Picard's iterative technique and the Itô formula of the conformable version is also established. In the present paper, we study the stability of solutions to (1.1). In Section 3, we prove the trivial solutions are stochastically stable, stochastically asymptotically stable, almost surely exponentially stable by setting suitable conditions on the constructed Lyapunov function. In Section 4, we propose the concepts of Ulam's type stability for (1.1) in the sense of probabilities and show the nontrivial solutions are almost surely Ulam's type stable. Two numerical simulation examples are presented in final section.

\section{PRELIMINARIES}

Definition 1 ( [2, Definition 2.1]). The conformable derivative with low index $\rho$ of a function $f:[\alpha, \infty) \rightarrow \mathbb{R}$ is defined as

$$
D_{\rho}^{\alpha} f(x)=\lim _{\varepsilon \rightarrow 0} \frac{f\left(x+\varepsilon(x-\alpha)^{1-\rho}\right)-f(x)}{\varepsilon}, \quad x>\alpha, \quad 0<\rho \leq 1 .
$$

Remark 1 ( [2, Theorem 2]). Fix $0<\rho \leq 1$ and $x>\alpha$. A function $f:[\alpha, \infty) \rightarrow$ $\mathbb{R}$ has a conformable derivative $D_{\rho}^{\alpha} f(x)$ if and only if it is differentiable at $x$ and $D_{\rho}^{\alpha} f(x)=(x-\alpha)^{1-\rho} f^{\prime}(x)$ holds.

Definition 2 ( $\left[7\right.$, Definition 4.1]). We say that an $\mathbb{R}^{n}$-valued stochastic process $X(\cdot)$ is a solution of $(1.1)$, if $X(\cdot)$ is continuous and $\mathbb{F}_{t}$-adapted and

$$
\begin{aligned}
& X(t)=X_{\alpha}+\int_{\alpha}^{t} b(X(\tau), \tau)(\tau-\alpha)^{\rho-1} d \tau \\
& +\int_{\alpha}^{t} \sigma(X(\tau), \tau)(\tau-\alpha)^{\rho-1} d W(\tau), t \in[\alpha, \alpha+h] .
\end{aligned}
$$

Lemma 1 ( [5, Theorem 3.8] ). (Doob's martingale inequalities) Let $\left\{M_{t}\right\}_{t \geq a}$ be an $\mathbb{R}^{n}$-valued martingale and $[a, b]$ an interval in $\mathbb{R}^{+}$. If $p \geq 1$ and $M_{t} \in \mathbb{L} p\left(\Omega, \mathbb{R}^{n}\right)$, then

$$
P\left\{\omega: \sup _{a \leq t \leq b}\left|M_{t}(\omega)\right| \geq c\right\} \leq \frac{1}{c^{p}} E\left|M_{b}\right|^{p}, \quad c>0 .
$$

Lemma 2 ([5, Lemma 5.4]). Denote $\mathcal{M}^{2}([a, b] ; \mathbb{R})$ the space of all real-valued measurable $\left\{\mathcal{F}_{t}\right\}$-adapted processes $g=\left\{g(t)_{a \leq t \leq b}\right\}$ such that

$$
E\left(\int_{a}^{b}|g(t)|^{2} d t\right)<\infty .
$$

If $g \in \mathbf{M}_{0}([a, b] ; \mathbb{R}) \subset \mathcal{M}^{2}([a, b] ; \mathbb{R})$, then, for $a \leq t \leq b$, we have

$$
E\left(\int_{a}^{b} g(t) d W(t)\right)=0
$$




$$
E\left(\left|\int_{a}^{b} g(t) d W(t)\right|^{2}\right)=E\left(\int_{a}^{b}|g(t)|^{2} d t\right) .
$$

Theorem 1 ( [7, Theorem 2.8]). Let $0<h<\infty, X(t), t \in[\alpha, \alpha+h]$ be an Itô process for

$$
D_{\rho}^{\alpha} X(t)=f(t)+g(t) \frac{d W(t)}{d t}, \rho \in(0,1]
$$

$Y(\cdot):=Y(X(\cdot), \cdot) \in C^{2,1}\left(\mathbb{R}^{n} \times[\alpha, \alpha+h], \mathbb{R}^{n}\right)$. Then, $Y(t), t \in[\alpha, \alpha+h]$ is an Itô process given by

$$
\begin{aligned}
& d Y(t)=\frac{\partial Y(X(t), t)}{\partial t} d t+\frac{\partial Y(X(t), t)}{\partial X} f(t)(t-\alpha)^{\rho-1} d t \\
& +\frac{\partial Y(X(t), t)}{\partial X} g(t)(t-\alpha)^{\rho-1} d W(t)+\frac{1}{2} \frac{\partial Y^{2}(X(t), t)}{\partial X^{2}} g^{2}(t)(t-\alpha)^{2 \rho-2} d t
\end{aligned}
$$

Definition 3 ( [5, Definition 2.1]). (i) The trivial solution of (1.1) is said to be stochastically stable or stable in probability if for every pair of $\varepsilon \in(0,1)$ and $r>0$, there exists a $\delta=\delta\left(\varepsilon, r, t_{0}\right)>0$, for all $t \geq t_{0}$ such that $P\{|X(t)|<r\} \geq 1-\varepsilon$, whenever $\left|X_{0}\right|<\delta$. Otherwise it is said to be stochastically unstable.

(ii) The trivial solution of (1.1) is said to be stochastically asymptotically stable if it is stochastically stable and, moreover, for every $\varepsilon \in(0,1)$, there exists a $\delta_{0}=$ $\delta_{0}\left(\varepsilon, t_{0}\right)>0$ such that $P\left\{\lim _{t \rightarrow \infty} X(t)=\mathbf{0}\right\} \geq 1-\varepsilon$, whenever $\left|X_{0}\right|<\delta_{0}$ and $\mathbf{0}$ denotes $n$-dimensional zero vector.

Definition 4 ([5, Definition 3.1]). The trivial solution of (1.1) is said to be almost surely exponentially stable, if $\lim _{t \rightarrow \infty} \sup \frac{1}{t} \ln |X(t)|<0$, a.s. for all $X_{0} \in \mathbb{R}^{n}$, where a.s. means almost surely.

Lemma 3 ([5, Lemma 2.4])). (Borel-Cantelli's lemma) If $\left\{A_{k}\right\} \subset \mathcal{F}$ and $\sum_{k=1}^{\infty} P\left(A_{k}\right)<\infty$. Then $P\left\{\lim _{k \rightarrow \infty} \sup A_{k}\right.$ i.o. $\}=0$, where i.o. means infinitely often.

Lemma $4\left(\left[8\right.\right.$, Theorem 1]). Set $x(\cdot), g(\cdot)$ is real continue function on $\left[t_{0}, t_{1}\right]$, $f(\cdot) \geq 0$ is the integrable function over interval $\left[t_{0}, t_{1}\right], g(\cdot) \geq 0$ is nondecreasing. If

$$
x(t) \leq g(t)+\int_{t_{0}}^{t} f(\tau) x(\tau) d \tau, \quad t \in\left[t_{0}, t_{1}\right]
$$

Then

$$
x(t) \leq g(t) \exp \left(\int_{t_{0}}^{t} f(\tau) d \tau\right), \quad t \in\left[t_{0}, t_{1}\right]
$$

\section{STABility OF TRIVIAL SOLUtions}

We first introduce the following assumptions and denote $|\cdot|$ by the norm of $\mathbb{R}^{n}$.

[H1] There exists a constant $L>0$ such that $b$ and $\sigma$ satisfying $|b(X, t)-b(\hat{X}, t)| \leq$ $L|X-\hat{X}|,|\sigma(X, t)-\sigma(\hat{X}, t)| \leq L|X-\hat{X}|, t \in[\alpha, \alpha+h], X, \hat{X} \in \mathbb{R}^{n}$. 
[H2] There exists a constant $L>0$ such that $b$ and $\sigma$ satisfying $|b(X, t)| \leq L \mid 1+$ $X|,| \sigma(X, t)|\leq L| 1+X \mid, t \in[\alpha, \alpha+h], X \in \mathbb{R}^{n}$.

[H3] $E\left(\left|X_{\alpha}\right|^{2}\right)<\infty$ where $X_{\alpha}$ is independent of $W^{+}(\alpha)$.

Lemma 5 ( [7, Theorem 4.3]). Suppose that [H1], [H2] and [H3] hold. Then, (1.1) has a unique solution $X(\cdot) \in \mathbb{L}_{n}^{2}[\alpha, \alpha+h]$ give by (2.1) provided that $\rho \in\left(\frac{1}{2}, 1\right]$.

Next, we discuss the stability of solutions to (1.1) under [H1], [H2] and [H3], which includes stability, asymptotical stability and almost surely exponentially stability.

For any $t \geq \alpha$, denote

$$
\begin{aligned}
& D_{\rho} V(X(t), t)=V_{t}(X(t), t)(t-\alpha)^{\rho-1}+V_{X}(X(t), t) b(X(t), t)(t-\alpha)^{\rho-1} \\
& +\frac{1}{2} \operatorname{trace}\left[\sigma^{T}(X(t), t) V_{X X}(X(t), t) \sigma(X(t), t)(t-\alpha)^{2 \rho-2}\right] .
\end{aligned}
$$

Set $k>0$ be arbitrary, denotes $S_{k}:=\left\{X(\cdot) \in \mathbb{R}^{n}|| X(\cdot) \mid<k\right\} . V(X(\cdot), \cdot) \in C^{2,1}\left(S_{k} \times\right.$ $\left.[\alpha, \infty) ; \mathbb{R}^{+}\right)$denote the family of all real-valued function $V(X(\cdot), \cdot)$ defined on $S_{k} \times$ $[\alpha, \infty)$ such that they are continuously twice differentiable in $X$ and once in $t . a \wedge b$ : the minimum of $a$ and $b, a \vee b:$ the maximum of $a$ and $b . I_{\{\cdot\}}$ is indicative function. Let $\mathbb{K}$ be the family of all continuous nondecreasing functions. $\mathbb{L}_{n}^{2}[\alpha, \alpha+h]$ is the space of $n$ dimensional $2 t h$ integrable functions defined on $[\alpha, \alpha+h]$.

We introduce assumptions for positive-definite function $V(X(\cdot), \cdot) \in C^{2,1}\left(S_{k} \times\right.$ $\left.[\alpha, \infty) ; \mathbb{R}^{+}\right)$.

[H4] $D_{\rho} V(X(\cdot), \cdot)$ is negative-definite, i.e. $D_{\rho} V(X(\cdot), \cdot) \leq 0$.

[H5] $V(X(\cdot), \cdot) \geq c_{1}|X(\cdot)|^{p}, c_{1}>1, p \geq 1$.

[H6] $D_{\rho} V(X(\cdot), \cdot) \leq c_{2} V(X(\cdot), \cdot), c_{2} \in \mathbb{R}$.

[H7] $\left|V_{X}(X(\cdot), \cdot) \sigma(X(\cdot), \cdot)(\cdot-\alpha)^{\rho-1}\right|^{2} \geq c_{3} V^{2}(X(\cdot), \cdot), c_{3}>0$.

Theorem 2. If [H1], [H2], [H3], [H4] hold and $\rho \in\left(\frac{1}{2}, 1\right]$, then the trivial solution of (1.1) is stochastically stable.

Proof. From Lemma 5, (1.1) has a unique solution $X(\cdot) \in \mathbb{L}_{n}^{2}[\alpha, \alpha+h]$. Following the same procedure as the proof of [5, p.111, Theorem 2.2], one can check the result. Here we present more details for the reader's convenience. Note that $V(X(t), t)$ is positive-definite and $V(\mathbf{0}, t) \equiv 0$, Then, there exists a function $\mu(\cdot) \in \mathbb{K}$, such that $V(X(t), t) \geq \mu(|X(t)|)$, for all $(X(t), t) \in\left(S_{k} \times[\alpha, \infty)\right)$.

Let $\varepsilon \in(0,1)$ and $r>0$ be arbitrary. Assume that $r<k$, since $V(X(\cdot), \cdot)$ is continuity and $V(\mathbf{0}, \alpha)=0$, we can find a $\delta=\delta(\varepsilon, r, \alpha)>0$ such that

$$
\frac{1}{\varepsilon} \sup _{X \in S_{\delta}} V(X(t), \alpha) \leq \mu(r) .
$$

Obviously, $\delta<r$, we fix initial value $X_{\alpha} \in S_{\delta}$ arbitrary, let $\tau$ be the first exit time of $X(t)$ from $S_{r}$, i.e. $\tau=\inf \left\{t>\alpha: X(t) \notin S_{r}\right\}$. 
By Theorem 1, for any $t>\alpha$, we obtain

$$
\begin{aligned}
& V(X(\tau \wedge t), \tau \wedge t) \\
& =V\left(X_{\alpha}, \alpha\right)+\int_{\alpha}^{\tau \wedge t} V_{s}(X(s), s)(s-\alpha)^{\rho-1} d s \\
& +\int_{\alpha}^{\tau \wedge t} V_{X}(X(s), s) b(X(s), s)(s-\alpha)^{\rho-1} d s \\
& +\int_{\alpha}^{\tau \wedge t} V_{X}(X(s), s) \sigma(X(s), s)(s-\alpha)^{\rho-1} d W(s) \\
& +\frac{1}{2} \operatorname{trace} \int_{\alpha}^{\tau \wedge t}\left[\sigma^{T}(X(s), s) V_{X X}(X(s), s) \sigma(X(s), s)(s-\alpha)^{2 \rho-2}\right] d s \\
& =V\left(X_{\alpha}, \alpha\right)+\int_{\alpha}^{\tau \wedge t} D_{\rho} V(X(s), s) d s \\
& +\int_{\alpha}^{\tau \wedge t} V_{X}(X(s), s) \sigma(X(s), s)(s-\alpha)^{\rho-1} d W(s) .
\end{aligned}
$$

By [H4], $D_{\rho} V(X(\cdot), \cdot) \leq 0$. Following the same procedure in the proof of [5, p.111, Theorem 2.2], taking the expectation on both sides, we have

$$
E V(X(\tau \wedge t), \tau \wedge t) \leq V\left(X_{\alpha}, \alpha\right) .
$$

Clearly $|X(\tau \wedge t)|=|X(\tau)|=r$ if $\tau<t$. Hence, we obtain

$$
E V(X(\tau \wedge t), \tau \wedge t) \geq E\left[I_{\{\tau \leq t\}} V(X(\tau), \tau)\right] \geq \mu(r) P\{\tau \leq t\} .
$$

Then, from (3.1), we get $P\{\tau \leq t\} \leq \varepsilon$. Letting $t \rightarrow \infty$, which implies $P\{\tau<\infty\} \leq \varepsilon$, i.e.

$$
P\{|X(t)| \leq r\} \geq 1-\varepsilon, t \geq \alpha .
$$

By Definition 3 (i), the trivial solution is stochastically stable. The proof is completed.

Theorem 3. If [H1], [H2], [H3] and [H4] hold, $V(X(\cdot), \cdot)$ is a decreasing function and $\rho \in\left(\frac{1}{2}, 1\right]$, then the trivial solution of $(1.1)$ is stochastically asymptotically stable.

Proof. From Theorem 2, the trivial solution of (1.1) is stochastic stable. Similarly, following the procedure of proof in [5, p.112, Theorem 2.3] and using Lemma 1, one can derive for any $\varepsilon \in(0,1)$, there exist a $\delta_{0}=\delta_{0}(\varepsilon, \alpha)>0$ such that $P\left\{\lim _{t \rightarrow \infty} X(t)=\right.$ 0 $\} \geq 1-\varepsilon$, whenever $\left|X_{\alpha}\right|<\delta_{0}$.

By Definition 3 (ii), the trivial solution is stochastically asymptotically stable.

Remark 2. For $\rho=1, \alpha=t_{0}$, Theorems 2 and 3 are the same as $[5, p .111$, Theorem 2.2] and [5, p.112, Theorem 2.3]. 
Theorem 4. If [H1], [H2], [H3], [H5], [H6] and [H7] hold and $\rho \in\left(\frac{1}{2}, 1\right]$, then, for all $X(t) \neq \mathbf{0}$ and $X_{\alpha} \neq \mathbf{0}$,

$$
\limsup _{t \rightarrow \infty} \frac{1}{t} \ln |X(t)| \leq \frac{c_{3}+2 c_{2}}{2 p}, \quad \text { a.s. }
$$

holds for all $X_{\alpha} \in \mathbb{R}^{n}$. In particular, if $c_{3}+2 c_{2}<0$, the trivial solution of equation (1.1) is almost surely exponentially stable.

Proof. By Theorem 1, we can deduce that for $\rho \in(0,1], t \geq \alpha$

$$
\begin{aligned}
& \ln V(X(t), t) \\
& \leq \ln V\left(X_{\alpha}, \alpha\right)+\int_{\alpha}^{t} \frac{\left(V_{s}(X(s), s)+V_{X}(X(s), s) b(X(s), s)\right)}{V(X(s), s)}(s-\alpha)^{\rho-1} d s \\
& +\int_{\alpha}^{t} \frac{V_{X}(X(s), s) \sigma(X(s), s)}{V(X(s), s)}(s-\alpha)^{\rho-1} d W(s) \\
& +\frac{1}{2} \int_{\alpha}^{t} \frac{\left|V_{X X}(X(s), s) V(X(s), s)-V_{X}(X(s), s) V_{X}(X(s), s)\right|}{V^{2}(X(s), s)}(d X(t))^{2} \\
& \leq \ln V\left(X_{\alpha}, \alpha\right)+\int_{\alpha}^{t} \frac{D_{\rho} V(X(s), s)}{V(X(s), s)} d s \\
& +\int_{\alpha}^{t} \frac{V_{X}(X(s), s) \sigma(X(s), s)}{V(X(s), s)}(s-\alpha)^{\rho-1} d W(s) \\
& -\frac{1}{2} \int_{\alpha}^{t} \frac{\left|V_{X}(X(s), s) \sigma(X(s), s)(s-\alpha)^{\rho-1}\right|^{2}}{V^{2}(X(s), s)} d s .
\end{aligned}
$$

Set $M(t):=\int_{\alpha}^{t} \frac{V_{X}(X(s), s) \sigma(X(s), s)}{V(X(s), s)}(s-\alpha)^{\rho-1} d W(s)$. Then, for any $\varepsilon \in(0,1)$ is arbitrarily, let $n=1,2 \ldots$, by [H7] and Lemma 1 , we can get

$$
\begin{aligned}
& P\left\{\sup _{\alpha \leq t \leq \alpha+n}\left[M(t)+\frac{\varepsilon}{2} \int_{\alpha}^{t} \frac{\left|V_{X}(X(s), s) \sigma(X(s), s)(s-\alpha)^{\rho-1}\right|^{2}}{V^{2}(X(s), s)} d s\right]>c_{3}(t-\alpha)\right\} \\
& \leq \frac{\varepsilon}{2 c_{3}(t-\alpha)} .
\end{aligned}
$$

Using Lemma 3, we have

$$
\begin{aligned}
& P\left\{\sup _{\alpha \leq t \leq \alpha+n}\left[M(t)+\frac{\varepsilon}{2} \int_{\alpha}^{t} \frac{\left|V_{X}(X(s), s) \sigma(X(s), s)(s-\alpha)^{\rho-1}\right|^{2}}{V^{2}(X(s), s)} d s\right]>c_{3}(t-\alpha) \text { i.o. }\right\} \\
& =0
\end{aligned}
$$

which implies

$$
M(t) \leq c_{3}(t-\alpha)-\frac{\varepsilon}{2} \int_{\alpha}^{t} \frac{\left|V_{X}(X(s), s) \sigma(X(s), s)\right|^{2}}{V^{2}(X(s), s)}(s-\alpha)^{2(\rho-1)} d s . \quad \text { a.s. }
$$


From [H6] and [H7], for $t \geq \alpha$, we have

$$
\ln V(X(t), t) \leq \ln V\left(X_{\alpha}, \alpha\right)+c_{2}(t-\alpha)+\frac{1}{2}\left[(1-\varepsilon) c_{3}(t-\alpha)\right] .
$$

Then, for $t \geq \alpha$

$$
\frac{1}{t} \ln V(X(t), t) \leq \frac{(t-\alpha)}{2 t}\left[(1-\varepsilon) c_{3}+2 c_{2}\right]+\frac{\ln V\left(X_{\alpha}, \alpha\right)}{t} .
$$

Thus, letting $t \rightarrow \infty$, we can get

$$
\lim _{t \rightarrow \infty} \sup \frac{1}{t} \ln V(X(t), t) \leq \frac{1}{2}\left[(1-\varepsilon) c_{3}+2 c_{2}\right] .
$$

Using [H5], we have

$$
\lim _{t \rightarrow \infty} \sup \frac{1}{t} \ln \left(c_{1}|X(t)|^{p}\right) \leq \lim _{t \rightarrow \infty} \frac{1}{t} \ln V(X(t), t) \leq \frac{1}{2}\left[(1-\varepsilon) c_{3}+2 c_{2}\right] .
$$

Note that $c_{1}>1, p \geq 1$, then

$$
\lim _{t \rightarrow \infty} \sup \frac{1}{t} \ln |X(t)|^{p}=p \lim _{t \rightarrow \infty} \frac{1}{t} \ln |X(t)| \leq \frac{1}{2}\left[(1-\varepsilon) c_{3}+2 c_{2}\right] .
$$

Finally, we obtain

$$
\lim _{t \rightarrow \infty} \sup \frac{1}{t} \ln |X(t)| \leq \frac{(1-\varepsilon) c_{3}+2 c_{2}}{2 p} .
$$

Since $\varepsilon$ is arbitrarily, we have

$$
\lim _{t \rightarrow \infty} \sup \frac{1}{t} \ln |X(t)| \leq \frac{c_{3}+2 c_{2}}{2 p} \text { a.s. }
$$

Then, if $c_{3}+2 c_{2}<0$, by Definition 4, the trivial solution of (1.1) is almost surely exponentially stable. The proof is completed.

Remark 3. Concerning on Theorem 4 for $\rho=1, \alpha=t_{0}$, [5, p.121, Theorem 3.3] uses different technique (exponential martingale inequality) in (3.2), i.e.

$$
P\left\{\sup _{t_{0} \leq t \leq t_{0}+n}\left[M(t)-\frac{\varepsilon}{2} \int_{t_{0}}^{t} \frac{\left|V_{X}(X(s), s) \sigma(X(s), s)\right|^{2}}{V^{2}(X(s), s)} d s\right]>\frac{2}{\varepsilon} \ln n\right\} \leq \frac{1}{n^{2}},
$$

then derives the following different inequality

$$
\lim _{t \rightarrow \infty} \sup \frac{1}{t} \ln |X(t)| \leq-\frac{c_{3}-2 c_{2}}{2 p} . \text { a.s. }
$$




\section{ULAM’S TYPE STABILITY}

Denote $C\left([\alpha, \alpha+h], \mathbb{R}^{+}\right)$by the family of all continuous functions from $[\alpha, \alpha+h]$ to $\mathbb{R}^{+}$. For $\forall \varepsilon>0, \varphi(\cdot) \in C\left([\alpha, \alpha+h], \mathbb{R}^{+}\right), t \in[\alpha, \alpha+h]$, consider

$$
\left|D_{\rho}^{\alpha} Y(t)-b(Y(t), t)-\sigma(Y(t), t) \frac{d W(t)}{d t}\right| \leq \varepsilon, \quad \frac{1}{2}<\rho \leq 1,
$$

and

$$
\left|D_{\rho}^{\alpha} Y(t)-b(Y(t), t)-\sigma(Y(t), t) \frac{d W(t)}{d t}\right| \leq \varepsilon \varphi(t), \frac{1}{2}<\rho \leq 1 .
$$

Definition 5. (1.1) is called almost surely Ulam-Hyers stable in the sense of probability, if there exists a constant $N>0$ such that for $\forall \varepsilon>0, r \in(0,1)$ and for each process $Y(\cdot) \in \mathbb{L}_{n}^{2}[\alpha, \alpha+h]$ satisfy

$$
P\{|Y(t)-X(t)| \leq N \varepsilon\} \geq 1-r, \quad t \in[\alpha, \alpha+h] .
$$

Remark 4. A process $Y(\cdot) \in \mathbb{L}_{n}^{2}[\alpha, \alpha+h]$ is a solution of (4.1), iff for $\forall \varepsilon>0$, there exists a function $G(\cdot) \in \mathbb{L}_{n}^{2}[\alpha, \alpha+h]$ such that $(i)|G(\cdot)|<\varepsilon^{2} ;(i i) D_{\rho}^{\alpha} Y(t)=$ $b(Y(t), t)+\sigma(Y(t), t) \frac{d W(t)}{d t}+G(t), t \in[\alpha, \alpha+h]$.

Definition 6. (1.1) is called almost surely Ulam-Hyers-Rassias stable in the sense of probability, if there exists a constant $\tilde{N}>0$ such that for $\forall \varepsilon>0, r \in(0,1)$, and $\varphi(\cdot) \in C\left([\alpha, \alpha+h], \mathbb{R}^{+}\right)$. for each process $Y(\cdot) \in \mathbb{L}_{n}^{2}[\alpha, \alpha+h]$ satisfy

$$
P\{|Y(t)-X(t)| \leq \tilde{N} \varepsilon \varphi(t)\} \geq 1-r, \quad t \in[\alpha, \alpha+h] .
$$

Remark 5. A process $Y(\cdot) \in \mathbb{L}_{n}^{2}[\alpha, \alpha+h]$ is a solution of (4.2), iff there exists a constant $M$ and function $\bar{G}(\cdot) \in \mathbb{L}_{n}^{2}[\alpha, \alpha+h]$ such that $(i)|\bar{G}(t)| \leq \varepsilon^{2} M \leq \varepsilon^{2} \varphi(t), t \in$ $[\alpha, \alpha+h] ;(i i) D_{\rho}^{\alpha} Y(t)=b(Y(t), t)+\sigma(Y(t), t) \frac{d W(t)}{d t}+\bar{G}(t), t \in[\alpha, \alpha+h]$.

Lemma 6. Let process $Y(\cdot)$ be a solution of equation (4.1) provided $\rho \in\left(\frac{1}{2}, 1\right]$. Then

$$
\begin{aligned}
& E\left(\mid Y(t)-Y_{\alpha}-\int_{\alpha}^{t} b(Y(\tau), \tau)(\tau-\alpha)^{\rho-1} d \tau\right. \\
& \left.-\left.\int_{\alpha}^{t} \sigma(Y(\tau), \tau)(\tau-\alpha)^{\rho-1} d W(\tau)\right|^{2}\right) \leq \frac{\varepsilon^{4} h^{2 \rho}}{2 \rho-1}
\end{aligned}
$$

hold for $t \in[\alpha, \alpha+h]$.

Proof. We know that $D_{\rho}^{\alpha} Y(t)=b(Y(t), t)+\sigma(Y(t), t) \frac{d W(t)}{d t}+G(t), t \in[\alpha, \alpha+h]$, with initial value $Y(\alpha)=Y_{\alpha}$, for $t \in[\alpha, \alpha+h]$, the solution can express as

$$
Y(t)=Y_{\alpha}+\int_{\alpha}^{t} b(Y(\tau), \tau)(\tau-\alpha)^{\rho-1} d \tau
$$




$$
+\int_{\alpha}^{t} \sigma(Y(\tau), \tau)(\tau-\alpha)^{\rho-1} d W(\tau)+\int_{\alpha}^{t} G(\tau)(\tau-\alpha)^{\rho-1} d \tau .
$$

Then, by Hölder inequality, for $t \in[\alpha, \alpha+h]$, we can get

$$
\begin{aligned}
& E\left(\mid Y(t)-Y_{\alpha}-\int_{\alpha}^{t} b(\tau, Y(\tau))(\tau-\alpha)^{\rho-1} d \tau\right. \\
& \left.-\left.\int_{\alpha}^{t} \sigma(\tau, Y(\tau))(\tau-\alpha)^{\rho-1} d W(\tau)\right|^{2}\right) \\
& =E\left|\int_{\alpha}^{t} G(\tau)(\tau-\alpha)^{\rho-1} d \tau\right|^{2} \\
& \leq\left. E\left|\int_{\alpha}^{t}\right| G(\tau)\right|^{2} d \tau \int_{\alpha}^{t}(\tau-\alpha)^{2(\rho-1)} d \tau \mid \\
& \leq E\left|\varepsilon^{4}(t-\alpha) \frac{(t-\alpha)^{2 \rho-1}}{2 \rho-1}\right| \\
& \leq E\left|\frac{\varepsilon^{4} h^{2 \rho}}{2 \rho-1}\right|=\frac{\varepsilon^{4} h^{2 \rho}}{2 \rho-1}, \rho \in\left(\frac{1}{2}, 1\right] .
\end{aligned}
$$

The proof is completed.

Lemma 7. Let the process $Y(\cdot)$ be a solution of equation (4.2) provided $\rho \in\left(\frac{1}{2}, 1\right]$. Then

$$
\begin{aligned}
& E\left(\mid Y(t)-Y_{\alpha}-\int_{\alpha}^{t} b(Y(\tau), \tau)(\tau-\alpha)^{\rho-1} d \tau\right. \\
& \left.-\left.\int_{\alpha}^{t} \sigma(Y(\tau), \tau)(\tau-\alpha)^{\rho-1} d W(\tau)\right|^{2}\right) \\
& \leq \frac{\varepsilon^{4} h^{2 \rho}}{2 \rho-1} \varphi^{2}(t)
\end{aligned}
$$

hold for $t \in[\alpha, \alpha+h]$.

Proof. We know that $D_{\rho}^{\alpha} Y(t)=b(t, Y(t))+\sigma(t, Y(t)) \frac{d W(t)}{d t}+\bar{G}(t), t \in[\alpha, \alpha+h]$, with initial value $Y(\alpha)=Y_{\alpha}$, for $t \in[\alpha, \alpha+h]$, the solution can expressed as

$$
\begin{aligned}
& Y(t)=Y_{\alpha}+\int_{\alpha}^{t} b(Y(\tau), \tau)(\tau-\alpha)^{\rho-1} d \tau \\
& +\int_{\alpha}^{t} \sigma(Y(\tau), \tau)(\tau-\alpha)^{\rho-1} d W(\tau)+\int_{\alpha}^{t} \bar{G}(\tau)(\tau-\alpha)^{\rho-1} d \tau .
\end{aligned}
$$

Then, by Hölder inequality, for $t \in[\alpha, \alpha+h]$, we can get

$$
E\left(\mid Y(t)-Y_{\alpha}-\int_{\alpha}^{t} b(Y(\tau), \tau)(\tau-\alpha)^{\rho-1} d \tau\right.
$$




$$
\begin{aligned}
& \left.-\left.\int_{\alpha}^{t} \sigma(Y(\tau), \tau)(\tau-\alpha)^{\rho-1} d W(\tau)\right|^{2}\right) \\
& =E\left(\left|\int_{\alpha}^{t} \bar{G}(\tau)(\tau-\alpha)^{\rho-1} d \tau\right|^{2}\right) \\
& \leq\left. E\left|\int_{\alpha}^{t}\right| G(\tau)\right|^{2} d \tau \cdot \int_{\alpha}^{t}(\tau-\alpha)^{2(\rho-1)} d \tau \mid \\
& \leq E\left|\varepsilon^{4} M^{2}(t-\alpha) \frac{(t-\alpha)^{2 \rho-1}}{2 \rho-1}\right| \\
& \leq E\left|\frac{\varepsilon^{4} h^{2 \rho}}{2 \rho-1} M^{2}\right|=\frac{\varepsilon^{4} h^{2 \rho}}{2 \rho-1} M^{2} .
\end{aligned}
$$

Noting $M \leq \varphi(\cdot)$, then $M^{2} \leq \varphi^{2}(\cdot)$. For $\alpha \leq t \leq \alpha+h$, we obtain

$$
\begin{aligned}
& E\left(\mid Y(t)-Y_{\alpha}-\int_{\alpha}^{t} b(Y(\tau), \tau)(\tau-\alpha)^{\rho-1} d \tau\right. \\
& \left.-\left.\int_{\alpha}^{t} \sigma(Y(\tau), \tau)(\tau-\alpha)^{\rho-1} d W(\tau)\right|^{2}\right) \leq \frac{\varepsilon^{4} h^{2 \rho}}{2 \rho-1} \varphi^{2}(t) .
\end{aligned}
$$

The proof is completed.

Theorem 5. Assume [H1], [H2] and [H3] hold and $\rho \in\left(\frac{1}{2}, 1\right]$. Then, (1.1) is almost surely Ulam-Hyers stable on $[\alpha, \alpha+h]$.

Proof. Let $Y(\cdot) \in \mathbb{L}_{n}^{2}[\alpha, \alpha+h]$ is a solution of (4.1), and $X(\cdot)$ is a solution of (1.1) give by (2.1). By Lemma 2, Lemma 6 and [H1], for $t \in[\alpha, \alpha+h]$, we can get

$$
\begin{aligned}
& E\left(|Y(t)-X(t)|^{2}\right) \\
& =E\left(\mid Y(t)-Y_{\alpha}-\int_{\alpha}^{t} b(Y(\tau), \tau)(\tau-\alpha)^{\rho-1} d \tau\right. \\
& -\int_{\alpha}^{t} \sigma(Y(\tau), \tau)(\tau-\alpha)^{\rho-1} d W(\tau) \\
& +\int_{\alpha}^{t}(b(Y(\tau), \tau)-b(Y(\tau), \tau))(\tau-\alpha)^{\rho-1} d \tau \\
& \left.+\left.\int_{\alpha}^{t}(\sigma(Y(\tau), \tau)-\sigma(Y(\tau), \tau))(\tau-\alpha)^{\rho-1} d W(\tau)\right|^{2}\right) \\
& \leq 3 \varepsilon^{4} \frac{h^{2 \rho}}{2 \rho-1}+3 L^{2}(t-\alpha) E\left(\int_{\alpha}^{t}|Y(\tau)-X(\tau)|^{2}(\tau-\alpha)^{2(\rho-1)} d \tau\right) \\
& +3 L^{2} E\left(\int_{\alpha}^{t}|Y(\tau)-X(\tau)|^{2}(\tau-\alpha)^{2(\rho-1)} d \tau\right) \\
& \leq 3 \varepsilon^{4} \frac{h^{2 \rho}}{2 \rho-1}+3 L^{2}(1+t-\alpha) \int_{\alpha}^{t} E\left(|Y(\tau)-X(\tau)|^{2}\right)(\tau-\alpha)^{2(\rho-1)} d \tau
\end{aligned}
$$




$$
\leq 3 \varepsilon^{4} \frac{h^{2 \rho}}{2 \rho-1}+3 L^{2}(1+h) \int_{\alpha}^{t} E\left(|Y(\tau)-X(\tau)|^{2}\right)(\tau-\alpha)^{2(\rho-1)} d \tau .
$$

Next, using Lemma 4 , for $t \in[\alpha, \alpha+h]$, we have

$$
\begin{aligned}
& E\left(|Y(t)-X(t)|^{2}\right) \leq 3 \varepsilon^{4} \frac{h^{2 \rho}}{2 \rho-1} \exp \left(3 L^{2}(1+h) \frac{(t-\alpha)^{2 \rho-1}}{2 \rho-1}\right) \\
& \leq 3 \varepsilon^{4} \frac{h^{2 \rho}}{2 \rho-1} \exp \left(3 L^{2}(1+h) \frac{h^{2 \rho-1}}{2 \rho-1}\right) \\
& \leq N \varepsilon^{4},
\end{aligned}
$$

where $N=3 \frac{h^{2 \rho}}{2 \rho-1} \exp \left(3 L^{2}(1+h) \frac{h^{2 \rho-1}}{2 \rho-1}\right)$.

Therefore, from Lemma 1, we can get

$$
P\left\{\sup _{\alpha \leq t \leq \alpha+h}|Y(t)-X(t)| \geq N \varepsilon\right\} \leq \frac{N \varepsilon^{4}}{(N \varepsilon)^{2}}=\frac{\varepsilon^{2}}{N} .
$$

By Lemma 3, we can get

$$
P\left\{\sup _{\alpha \leq t \leq \alpha+h}|Y(t)-X(t)| \geq N \varepsilon \text { i.o. }\right\}=0 . \quad \text { a.s. }
$$

Then, for $\forall r \in(0,1)$, we have

$$
P\left\{\sup _{\alpha \leq t \leq \alpha+h}|Y(t)-X(t)| \leq N \varepsilon\right\} \geq 1-r .
$$

From Definition 5, (1.1) is almost surely Ulam-Hyers stable. The proof is completed.

Theorem 6. Assume [H1], [H2], [H3] hold, $\varphi(\cdot)$ is nondecreasing and $\rho \in\left(\frac{1}{2}, 1\right]$. Then (1.1) is almost surely Ulam-Hyers-Rassias stable on $[\alpha, \alpha+h]$.

Proof. Let $Y(\cdot) \in \mathbb{L}_{n}^{2}[\alpha, \alpha+h]$ is a solution of (4.2), $X(\cdot)$ is a solution of (1.1) given by (2.1). By Lemma 2, Lemma 7 and [H1], for $t \in[\alpha, \alpha+h]$, we have

$$
\begin{aligned}
& E\left(|Y(t)-X(t)|^{2}\right) \\
& =E\left(\mid Y(t)-Y_{\alpha}-\int_{\alpha}^{t} b(Y(\tau), \tau)(\tau-\alpha)^{\rho-1} d \tau\right. \\
& -\int_{\alpha}^{t} \sigma(Y(\tau), \tau)(\tau-\alpha)^{\rho-1} d W(\tau) \\
& +\int_{\alpha}^{t}(b(Y(\tau), \tau)-b(Y(\tau), \tau))(\tau-\alpha)^{\rho-1} d \tau \\
& \left.+\left.\int_{\alpha}^{t}(\sigma(Y(\tau), \tau)-\sigma(Y(\tau), \tau))(\tau-\alpha)^{\rho-1} d W(\tau)\right|^{2}\right) \\
& \left.\leq 3 \frac{\varepsilon^{4} h^{2 \rho}}{2 \rho-1} \varphi^{2}(t)+3 L^{2}(t-\alpha) \int_{\alpha}^{t} E|Y(\tau)-X(\tau)|^{2}(\tau-\alpha)^{2(\rho-1)} d \tau\right)
\end{aligned}
$$




$$
\begin{aligned}
& +3 L^{2} \int_{\alpha}^{t} E|Y(\tau)-X(\tau)|^{2}(\tau-\alpha)^{2(\rho-1)} d \tau \\
& \leq 3 \frac{\varepsilon^{4} h^{2 \rho}}{2 \rho-1} \varphi^{2}(t)+3 L^{2}(1+t-\alpha) \int_{\alpha}^{t} E|Y(\tau)-X(\tau)|^{2}(\tau-\alpha)^{2(\rho-1)} d \tau \\
& \leq 3 \frac{\varepsilon^{4} h^{2 \rho}}{2 \rho-1} \varphi^{2}(t)+3 L^{2}(1+h) \int_{\alpha}^{t} E|Y(\tau)-X(\tau)|^{2}(\tau-\alpha)^{2(\rho-1)} d \tau .
\end{aligned}
$$

Note $\varphi(\cdot)$ is nondecreasing and $\left(\varphi^{2}\right)^{\prime}=2 \varphi \varphi^{\prime} \geq 0$. From Lemma 4 , for $t \in[\alpha, \alpha+$ $h]$, we have

$$
\begin{aligned}
& E\left(|Y(t)-X(t)|^{2}\right) \leq 3 \frac{\varepsilon^{4} h^{2 \rho}}{2 \rho-1} \varphi^{2}(t) \exp \left(3 L^{2}(1+h) \int_{\alpha}^{t}(\tau-\alpha)^{2(\rho-1)} d \tau\right) \\
& \leq 3 \frac{\varepsilon^{4} h^{2 \rho}}{2 \rho-1} \varphi^{2}(t) \exp \left(3 L^{2}(1+h) \frac{(t-\alpha)^{2 \rho-1}}{2 \rho-1}\right) \\
& \leq \tilde{N} \varepsilon^{4} \varphi^{2}(t),
\end{aligned}
$$

where $\tilde{N}=3 \frac{h^{2 \rho}}{2 \rho-1} \exp \left(3 L^{2}(1+h) \frac{h^{2 \rho-1}}{2 \rho-1}\right)$. Therefore, by Lemma 1, we have

$$
P\left\{\sup _{\alpha \leq t \leq \alpha+h}|Y(t)-X(t)| \geq \tilde{N} \varepsilon \varphi(t)\right\} \leq \frac{\tilde{N} \varepsilon^{4} \varphi^{2}(t)}{\tilde{N}^{2} \varepsilon^{2} \varphi^{2}(t)}=\frac{\varepsilon^{2}}{\tilde{N}} .
$$

By Lemma 3, we get

$$
P\left\{\sup _{\alpha \leq t \leq \alpha+h}|Y(t)-X(t)| \geq \tilde{N} \varepsilon \varphi(t) \text { i.o. }\right\}=0 . \quad \text { a.s. }
$$

Then, for $\forall r \in(0,1)$, we have

$$
P\left\{\sup _{\alpha \leq t \leq \alpha+h}|Y(t)-X(t)| \leq \tilde{N} \varepsilon \varphi(t)\right\} \geq 1-r .
$$

From Definition 6, (1.1) is almost surely Ulam-Hyers stable. The proof is completed.

\section{EXAMPLES}

Example 1. Consider an one-dimensional stochastic differential equation

$$
\left\{\begin{aligned}
D_{\rho}^{\alpha} X(t) & =b(X(t), t)+\sigma(X(t), t) \frac{d W(t)}{d t}, t \geq \alpha, \rho \in\left(\frac{1}{2}, 1\right], \\
X(\alpha) & =X_{\alpha},
\end{aligned}\right.
$$

where $X_{\alpha} \in \mathbb{R}$ and $X_{\alpha} \neq \pm \infty, b: \mathbb{R} \times[\alpha, \infty) \rightarrow \mathbb{R}, \sigma: \mathbb{R} \times[\alpha, \infty) \rightarrow \mathbb{R}$.

Let $b, \sigma$ satisfying [H1], [H2] and

$$
b(X, \cdot)=a(\cdot) X(\cdot), \quad \sigma(X, \cdot)=b(\cdot) X(\cdot),
$$


in a neighbourhood of $X(\cdot)=0$, where $a(\cdot), b(\cdot)$ are bounded Borel-measurable functions. Assume there is a pair of positive constants $\lambda$ and $K$ such that

$$
-K \leq \int_{\alpha}^{t}\left(a(\tau)+\frac{\varepsilon-1}{2} b^{2}(\tau)(\tau-\alpha)^{\rho-1}+\lambda\right) d \tau \leq K, t \geq \alpha .
$$

We define the stochastic Lyapunov function

$$
V(X, t)=|X(t)|^{\varepsilon} \exp \left[-\varepsilon \int_{\alpha}^{t}\left(a(\tau)+\frac{\varepsilon-1}{2} b^{2}(\tau)(\tau-\alpha)^{\rho-1}+\lambda\right) d \tau\right], t \geq \alpha .
$$

From (5.3), for $t \geq \alpha$, we have

$$
|X(t)|^{\varepsilon} e^{-\varepsilon K} \leq V(X, t) \leq|X(t)|^{\varepsilon} e^{\varepsilon K} .
$$

Hence, $V$ is positive-define. Combing (5.2) and (5.4), for $t \geq \alpha$, we have

$$
\begin{aligned}
& D_{\rho} V(X(t), t)=|X(t)|^{\varepsilon} \exp \left[-\varepsilon \int_{\alpha}^{t}\left(a(\tau)-\frac{\varepsilon-1}{2} b^{2}(\tau)(\tau-\alpha)^{\rho-1}+\lambda\right) d \tau\right] \\
& \cdot-\varepsilon\left(a(t)+\frac{\varepsilon-1}{2} b^{2}(t)(t-\alpha)^{\rho-1}+\lambda\right)(t-\alpha)^{\rho-1} \\
& +\varepsilon|X(t)|^{\varepsilon-1} \exp \left[-\varepsilon \int_{\alpha}^{t}\left(a(\tau)+\frac{\varepsilon-1}{2} b^{2}(\tau)(\tau-\alpha)^{\rho-1}+\lambda\right) d \tau\right] \\
& \cdot[a(t) X(t)](t-\alpha)^{\rho-1} \\
& +\frac{1}{2}\left(b^{T}(t) X(t) \varepsilon(\varepsilon-1)|X(t)|^{\varepsilon-2} X(t) b(t)\right) \\
& \cdot \exp \left[-\varepsilon \int_{\alpha}^{t}\left(a(\tau)+\frac{\varepsilon-1}{2} b^{2}(\tau)(\tau-\alpha)^{\rho-1}+\lambda\right) d \tau\right](t-\alpha)^{2(\rho-1)} \\
& \leq-\varepsilon \lambda e^{-\varepsilon K}|X(t)|^{\varepsilon}(t-\alpha)^{\rho-1} \\
& <0 .
\end{aligned}
$$

From above [H4] holds. By Theorem 2, the trivial solution of (5.1) is stochastically stable under (5.2) and (5.3).

Example 2. Consider the following conformable stochastic differential equations and give the simulation results of the Ulam's type stable of the solution.

$$
\left\{\begin{aligned}
D_{\rho}^{\alpha} X(t) & =r X(t)+\beta(t-\alpha)^{1-\rho} X(t) \frac{d W(t)}{d t}, t \in[\alpha, \alpha+h] \\
X(\alpha) & =X_{\alpha} \neq \pm \infty
\end{aligned}\right.
$$

The solution of (5.5) can express as

$$
X(t)=X_{\alpha} e^{\left(r \frac{(t-\alpha)^{\rho}}{\rho}+\beta W(t)-\frac{1}{2} \beta^{2}(t-\alpha)\right)}, t \in[\alpha, \alpha+h] .
$$


Set $X_{0}=10, r=0.12, \beta=0.18, \alpha=0, h=500$, Brownian motion $W(t)$ and its white noise $\frac{d W(t)}{d t}$ when $t \in[0,500]$. Then (5.6) reduces to

$$
X(t)=10 e^{\left(0.12 \frac{t^{\rho}}{\rho}+0.18 W(t)-0.0072 t\right)}, t \in[0,500] .
$$

Let $\varphi(t)=\exp \left(0.5 \frac{t^{\rho}}{\rho}\right), t \in[0,500]$, we consider

$$
\left|D_{\rho}^{\alpha} X(t)-r X(t)-\beta t^{1-\rho} X(t) \frac{d W(t)}{d t}\right| \leq \varepsilon, \frac{1}{2}<\rho \leq 1, t \in[0,500],
$$

and

$$
\left|D_{\rho}^{\alpha} X(t)-r X(t)-\beta t^{1-\rho} X(t) \frac{d W(t)}{d t}\right| \leq \varepsilon \varphi(t), \frac{1}{2}<\rho \leq 1, t \in[0,500] .
$$

Clearly, (5.5) is Ulam-Hyers stable and Ulam-Hyers-Rassias stable from Theorems 5 and 6 , respectively.

\section{REFERENCES}

[1] R. Khalila , M. AlHorani , A. Yousef, and M. Sababheh, "A new definition of fractional derivative." J. Comput. Appl. Math. , vol. 264, pp. 65-70, 2014, doi: 10.1016/j.cam.2014.01.002.

[2] T. Abdeljawad , "On conformable fractional calculus." J. Comput. Appl. Math., vol. 279, pp. 57-66, 2015, doi: 10.1016/j.cam.2014.10.016.

[3] W. Chung, "Fractional Newton mechanics with conformable fractional derivative." J. Comput. Appl. Math. , vol. 290, pp. 150-158, 2015, doi: 10.1016/j.cam.2015.04.049.

[4] T. U. Khan and M. A. Khan, "Generalized conformable fractional operators." J. Comput. Appl. Math. , vol. 346, pp. 378-389, 2019, doi: 10.1016/j.cam.2018.07.018.

[5] X. Mao, Stochastic differential equations and application. Horwood Publishing Limited, 2007.

[6] B. Øksendal, Stochastic differential equations an introduction with application. Springer-Verlag, 2000.

[7] G. Xiao, J. Wang, and D. O'Regan, "Existence, uniqueness and continuous dependence of solutions to conformable stochastic differential equations." submitted, 2019.

[8] H. Ye, J. Gao, and Y. Ding, " A generalized Gronwall inequality and its application to a fractional differential equation." J. Math. Anal. Appl., vol. 328, pp. 1075-1081, 2007, doi: 10.1016/j.jmaa.2006.05.061.

[9] D. Zhao, X. Pan, and M. Luo, "A new framework for multivariate general conformable fractional calculus and potential applications." Physica A-Stat. Mech. Appl., vol. 510, no. 10.1016/j.physa.2018.06.070, pp. 271-280, 2018.

Authors' addresses

Guanli Xiao

JinRong Wang, Department of Mathematics, Guizhou University, Guiyang, Guizhou 550025, P.R. China, and Qufu Normal University

E-mail address: glxiaomath@126.com 


\section{JinRong Wang}

JinRong Wang, Department of Mathematics, Guizhou University, Guiyang, Guizhou 550025, P.R. China, and Qufu Normal University, School of Mathematical Sciences, Qufu, Shandong 273165, P.R. China

E-mail address: jrwangegzu.edu.cn 